\title{
The average order of the Möbius function for Beurling primes
}

Article

Accepted Version

Neamah, A. A. and Hilberdink, T. W. (2019) The average order of the Möbius function for Beurling primes. International Journal of Number Theory, 16 (5). pp. 1005-1011. ISSN 17937310 doi: https://doi.org/10.1142/s1793042120500517 Available at https://centaur.reading.ac.uk/88220/

It is advisable to refer to the publisher's version if you intend to cite from the work. See Guidance on citing.

To link to this article DOI: http://dx.doi.org/10.1142/s1793042120500517

Publisher: World Scientific Publishing

All outputs in CentAUR are protected by Intellectual Property Rights law, including copyright law. Copyright and IPR is retained by the creators or other copyright holders. Terms and conditions for use of this material are defined in the End User Agreement.

\section{www.reading.ac.uk/centaur}

\section{CentAUR}

Central Archive at the University of Reading

Reading's research outputs online 


\title{
The average order of the Möbius function for Beurling primes
}

\author{
Ammar Ali Neamah ${ }^{1,2}$ and Titus W Hilberdink ${ }^{1}$ \\ ${ }^{1}$ Department of Mathematics, University of Reading, Whiteknights, PO Box 220, Reading RG6 6AX, \\ UK; t.w.hilberdink@reading.ac.uk \\ ${ }^{2}$ Faculty of Computer Science and Mathematics, University of Kufa, Najaf, Iraq; \\ ammara.meamah@uokufa.edu.iq, a.a.n.al-rammahi@pgr.reading.ac.uk
}

\begin{abstract}
In this paper, we study the counting functions $\psi_{\mathcal{P}}(x), N_{\mathcal{P}}(x)$ and $M_{\mathcal{P}}(x)$ of a generalized prime system $\mathcal{N}$. Here $M_{\mathcal{P}}(x)$ is the partial sum of the Möbius function over $\mathcal{N}$ not exceeding $x$. In particular, we study these when they are asymptotically well-behaved, in the sense that $\psi_{\mathcal{P}}(x)=x+O\left(x^{\alpha+\varepsilon}\right), N_{\mathcal{P}}(x)=\rho x+O\left(x^{\beta+\varepsilon}\right)$ and $M_{\mathcal{P}}(x)=O\left(x^{\gamma+\varepsilon}\right)$, for some $\rho>0$ and $\alpha, \beta, \gamma<1$. We show that the two largest of $\alpha, \beta, \gamma$ must be equal and at least $\frac{1}{2}$.
\end{abstract}

2010 AMS Mathematics Subject Classification: 11N80, 11N56

Keywords and phrases: Beurling's generalized primes, Möbius function

\section{Introduction}

A Beurling generalized prime system $\mathcal{P}$ is an unbounded sequence of real numbers $p_{1}, p_{2}, p_{3}, \ldots$ satisfying

$$
1<p_{1} \leq p_{2} \leq \cdots \leq p_{n} \leq \cdots .
$$

We call these numbers generalized primes (or $g$-primes), and from them we form the system $\mathcal{N}$ of generalized integers (or $g$-integers) associated to $\mathcal{P}$. These are the numbers of the form

$$
p_{1}^{\alpha_{1}} p_{2}^{\alpha_{2}} \cdots p_{k}^{\alpha_{k}}
$$

where $\alpha_{1}, \ldots, \alpha_{k} \in \mathbb{N}_{0}$. In other words, $\mathcal{N}$ (viewed as a multi-set) is the semi-group generated by the (multi-set) $\mathcal{P}$ under multiplication. Such systems were first defined and investigated by Beurling [1] in 1937 and have been studied by many researchers since then (see for instance [2], [5] and the numerous references therein). Attached to these systems are the counting functions

$$
\pi_{\mathcal{P}}(x)=\sum_{\substack{p \leq x \\ p \in \mathcal{P}}} 1, \quad N_{\mathcal{P}}(x)=\sum_{\substack{n \leq x \\ n \in \mathcal{N}}} 1, \quad \psi_{\mathcal{P}}(x)=\sum_{\substack{p^{k} \leq x \\ p \in \mathcal{P} \\ k \in \mathbb{N}}} \log p
$$

which generalize the usual counting functions. In each case, the sum is over all possible elements from the multi-set $\mathcal{P}$ or $\mathcal{N}$ with the given constraint. We are also interested in the generalized Möbius function defined to be $\mu_{\mathcal{P}}(1)=1, \mu_{\mathcal{P}}\left(p_{i_{1}} \cdots p_{i_{k}}\right)=(-1)^{k}$ for distinct $g$-primes (i.e. $i_{1}, \ldots, i_{k}$ are distinct) and zero otherwise. Strictly speaking this need not be a function if two such products are numerically the same. In any case, we define the sum function

$$
M_{\mathcal{P}}(x)=\sum_{\substack{n \leq x \\ n \in \mathcal{N}}} \mu_{\mathcal{P}}(n)
$$


This generalizes the usual $M(x)=\sum_{n \leq x} \mu(n)$. The associated Beurling zeta function is defined as usual by

$$
\zeta_{\mathcal{P}}(s)=\prod_{p \in \mathcal{P}} \frac{1}{1-\frac{1}{p^{s}}}=\sum_{n \in \mathcal{N}} \frac{1}{n^{s}} .
$$

We are interested in systems for which one or more of $\psi_{\mathcal{P}}(x)-x, N_{\mathcal{P}}(x)-\rho x$, or $M_{\mathcal{P}}(x)$ is $O\left(x^{\theta}\right)$ for some $\theta<1$ (and $\rho>0$ ). More precisely, we define three numbers $\alpha, \beta, \gamma$ by the following:

$$
\begin{aligned}
\psi_{\mathcal{P}}(x) & =x+O\left(x^{\alpha+\varepsilon}\right) \\
N_{\mathcal{P}}(x) & =\rho x+O\left(x^{\beta+\varepsilon}\right) \\
M_{\mathcal{P}}(x) & =O\left(x^{\gamma+\varepsilon}\right)
\end{aligned}
$$

hold for all $\varepsilon>0$ but no $\varepsilon<0$. For example, for $\mathcal{N}=\mathbb{N}, \beta=0$ while $\alpha=\gamma \geq \frac{1}{2}$ due to the Riemann zeros. At the outset we are only interested in those systems for which the abscissa of convergence of the Dirichlet series for $\zeta_{\mathcal{P}}$ is 1 . Thus $\alpha, \beta, \gamma \in[0,1]$ in any case.

For (1) and (2) to hold simultaneously for some $\alpha, \beta<1$ is akin to having a kind of Riemann Hypothesis being true for such a system. In [11], it was shown that such a system does exist with $\alpha, \beta \leq \frac{1}{2}$. On the other hand, in [6], it was shown that it is impossible to have both $\alpha$ and $\beta$ less than $\frac{1}{2}$.

We note that (3) is related to an interesting problem in its own right: how small can $M_{\mathcal{P}}(x)$ be made for a system with abscissd 1 equal to 1? In other words, how much cancellation can occur in the sum for $M_{\mathcal{P}}(x)$ ? Of course, for $\mathcal{N}=\mathbb{N}, M(x)=\Omega(\sqrt{x})$ on account of the Riemann zeros, but without this knowledge it is not clear how to even prove $M(x)=\Omega\left(x^{a}\right)$ for some $a>0$. This is similar to a question of Kahane and Saias [9] who ask how small $\sum_{n \leq x} f(n)$ can be for $f$ completely multiplicative.

It is also related to the more general question of the size of $M_{\mathcal{P}}(x)$ and how it relates to the other functions. For example, much work has been done to determine under what conditions one has $M_{\mathcal{P}}(x)=o(x)$ (see for example Chapter 14 of [5]). Zhang [10] was the first to note that $\mathrm{PNT}$ is not equivalent to $M_{\mathcal{P}}(x)=o(x)$. For the most general results giving $M_{\mathcal{P}}(x)=o(x)$, see the very recent papers [3] and [4].

Our main result is the following:

\section{Theorem 1}

Of the numbers $\alpha, \beta, \gamma$, the two largest must be the same and at least $\frac{1}{2}$.

This result implies that for $M_{\mathcal{P}}(x)=O\left(x^{\gamma}\right)$ with $\gamma<\frac{1}{2}$ to hold, we need the system to have somewhat chaotic $g$-primes and $g$-integers; i.e. the errors in (1) and (2) have to be $\Omega\left(x^{\frac{1}{2}-\varepsilon}\right)$ for every $\varepsilon>0$. It may be conjectured that having $\gamma<\frac{1}{2}$ is actually impossible.

\section{Some Relevant Results}

In order to prove the main result we shall need some relevant notions as well as existing results about $g$-prime systems.

Let $f(s)=\sum_{n=1}^{\infty} \frac{a_{n}}{b_{n}^{s}}$ be a generalized Dirichlet series where $b_{n}>0$ is strictly increasing with finite abscissae of absolute convergence $\sigma_{a}$. Suppose $f$ has a meromorphic continuation to $H_{\alpha}:=\{s \in \mathbb{C}: \Re s>\alpha\}$. We say $f$ has finite order in $H_{\alpha}$ if

$$
f(\sigma+i t) \ll|t|^{\lambda} \quad(|t| \geq 1)
$$

\footnotetext{
${ }^{1}$ The abscissa of convergence of $\zeta_{\mathcal{P}}(s)$. With abscissa $\sigma_{c}$, we trivially have $M_{\mathcal{P}}(x) \ll x^{\sigma_{c}+\varepsilon}$. Without the condition on the abscissa, $M_{\mathcal{P}}(x)$ can even be bounded: take $\mathcal{P}=\left\{2^{2^{n}}: n \in \mathbb{N}_{0}\right\}$. Then $M_{\mathcal{P}}(x)=0,1$ or -1 .
} 
for $\sigma>\alpha$. As such, we can define the Lindelöf function $\mu_{f}(\sigma)$ to be the infimum of such $\lambda$. It is well-known that $\mu_{f}$ is non-negative, decreasing and convex and for $\sigma>\sigma_{a}, \mu_{f}(\sigma)=0$.

The following result about such Dirichlet series and "counting function"

$$
A(x):=\sum_{b_{n} \leq x} a_{n}
$$

was essentially proved in [6], Proposition 3 (see also [8], Theorem 2.1). It was proven for the case where $a_{n} \geq 0$ such that $a_{n} \ll n^{\varepsilon}$ for all $\varepsilon>0$. This latter condition however is not necessary. Also we shall require a particular case when $a_{n}$ is also sometimes negative.

\section{Theorem A}

Let $f(s)=\sum_{n=1}^{\infty} \frac{a_{n}}{b_{n}^{s}}$ have abscissa of convergence $\sigma_{c} \leq 1$. Suppose that for some $\alpha \in[0,1)$ and $c \in \mathbb{C}$, we have

$$
A(x)=c x+O\left(x^{\alpha+\varepsilon}\right) \quad \text { for all } \varepsilon>0 .
$$

Then $f(s)$ has an analytic continuation to $H_{\alpha} \backslash\{1\}$ with a simple pole at $s=1$ with residu@ $c$ and $f$ has finite order; indeed $\mu_{f}(\sigma) \leq 1$ for $\sigma>\alpha$.

Conversely, suppose that for some $\alpha \in[0,1), f(s)$ has an analytic continuation to $H_{\alpha}$ except for a simple pole at $s=1$ with residue c. Further assume that $\mu_{f}(\sigma)=0$ for $\sigma>\alpha$ and either (i) $a_{n} \geq 0$ or

$$
\sum_{x-1<b_{n} \leq x}\left|a_{n}\right|=O\left(x^{\alpha+\varepsilon}\right) \quad \text { for all } \varepsilon>0
$$

Then (4) holds.

Proof. The proof of the first part is standard and follows on writing

$$
f(s)=s \int_{1}^{\infty} \frac{A(x)}{x^{s+1}} d x=\frac{c s}{s-1}+s \int_{1}^{\infty} \frac{A(x)-c x}{x^{s+1}} d x
$$

and noting that the integral on the right converges absolutely to a holomorphic function on $H_{\alpha}$.

For the converse, we follow the proof of Proposition 3 in [6] as much as possible. This leads to

$$
A(x)-c x \ll \frac{x}{T^{1-\varepsilon}}+x^{\alpha+\varepsilon} T^{\varepsilon}+\frac{x^{1+\varepsilon}}{T}+\frac{x}{T} \sum_{\frac{x}{2}<b_{n}<2 x} \frac{\left|a_{n}\right|}{\left|b_{n}-x\right|}
$$

for every $T>1$ and $\varepsilon>0-$ see equation $(3.7)$ of $[6]$.

Now, as in [6], consider $x$ such that

$$
\left(x-\frac{1}{x^{2}}, x+\frac{1}{x^{2}}\right) \cap\left\{b_{k}: k \in \mathbb{N}\right\}=\emptyset .
$$

For such $x,\left|b_{n}-x\right| \geq \frac{1}{x^{2}}$ for all $n$ and the sum on the right in (6) is at most

$$
x^{2} \sum_{\frac{x}{2}<b_{n}<2 x}\left|a_{n}\right| .
$$

In case (i), this is $O\left(x^{3+\varepsilon}\right)$, while in case (ii), it is $O\left(x^{3+\alpha+\varepsilon}\right)$ by (5).

Taking $T=x^{4}$ in (6) shows that (4) holds whenever $x \rightarrow \infty$ satisfying (7). As shown in [8] (see (2.3)), for every $x$ there exist $x_{1} \in(x-1, x), x_{2} \in(x, x+1)$ such that $x_{1}, x_{2}$ satisfy $(7)$. Thus (4) holds for $x_{1}$ and $x_{2}$.

\footnotetext{
${ }^{2}$ Of course, if $c=0$, the pole is removable.
} 
For case (i), positivity of $a_{n}$ implies $A\left(x_{1}\right) \leq A(x) \leq A\left(x_{2}\right)$. Hence (4) follows for $x$.

For case (ii), we use (5). We have

$$
\left|A(x)-A\left(x_{1}\right)\right| \leq \sum_{x-1<b_{n} \leq x}\left|a_{n}\right| \ll x^{\alpha+\varepsilon}
$$

by (5). Hence (4) follows.

We also require the following result from [8] (Theorem 2.3).

\section{Theorem B}

Suppose (1) and (2) hold for some $\alpha, \beta<1$. Then for $\sigma>\Theta:=\max \{\alpha, \beta\}$ and uniformly for $\sigma \geq \Theta+\delta($ any $\delta>0)$,

$$
\frac{\zeta_{\mathcal{P}}^{\prime}(\sigma+i t)}{\zeta_{\mathcal{P}}(\sigma+i t)}=O\left((\log |t|)^{\frac{1-\sigma}{1-\Theta}+\varepsilon}\right) \quad \text { and } \quad \zeta_{\mathcal{P}}(\sigma+i t), \frac{1}{\zeta_{\mathcal{P}}(\sigma+i t)}=O\left(\exp \left\{(\log |t|)^{\frac{1-\sigma}{1-\Theta}+\varepsilon}\right\}\right)
$$

for all $\varepsilon>0$. In particular, for $\sigma>\Theta$, the Lindelöf functions for $\zeta_{\mathcal{P}}$ and $\frac{1}{\zeta_{\mathcal{P}}}$ are zero.

Actually, the statement of Theorem 2.3 in [8] does not mention $\frac{1}{\zeta_{\mathcal{P}}}$ but the proof, which argues from $\log \zeta_{\mathcal{P}}$ clearly applies also to $-\log \zeta_{\mathcal{P}}=\log \frac{1}{\zeta_{\mathcal{P}}}$.

Also, we have the following two consequences as described at the end of section 2 in $[8]$ :

(a) If $\alpha>\beta$, then $\zeta_{\mathcal{P}}$ has infinitely many zeros on, or arbitrarily close to, the line $\sigma=\alpha$.

(b) If $\alpha<\beta$, then $\zeta_{\mathcal{P}}$ and $\frac{1}{\zeta_{\mathcal{P}}}$ have infinite order in the strip $\{s \in \mathbb{C}: \alpha<\Re s<\beta\}$.

\section{Proof of Theorem 1}

Let $\Theta:=\max \{\alpha, \beta\}$. We use the converse part of Theorem A with $f(s)=\frac{1}{\zeta_{\mathcal{P}}(s)}$. This function has an analytic continuation to $H_{\Theta}$ and, by Theorem $\mathrm{B}$, has zero order here. Further, $A(x)=$ $M_{\mathcal{P}}(x)$ and

$$
\sum_{\substack{x \rightarrow 1<n \leq x \\ n \in \mathcal{N}}}\left|\mu_{\mathcal{P}}(n)\right| \leq N_{\mathcal{P}}(x)-N_{\mathcal{P}}(x-1) \ll x^{\beta+\varepsilon} \leq x^{\Theta+\varepsilon} .
$$

Thus (5), and hence (4), holds (with $c=0$ ). That is, $M_{\mathcal{P}}(x)=O\left(x^{\Theta+\varepsilon}\right)$; i.e. $\gamma \leq \Theta$.

Now suppose $\alpha>\beta$. Then $\zeta_{\mathcal{P}}$ has infinitely many zeros on, or arbitrarily close to, the line $\sigma=\alpha$. Thus $\gamma \geq \alpha-\delta$ for any $\delta>0$; i.e. $\gamma \geq \alpha$ and so $\gamma=\alpha$.

Now suppose $\alpha<\beta$. Then the Lindelöf functions for $\zeta_{\mathcal{P}}$ and $1 / \zeta_{\mathcal{P}}$ are infinite for $\sigma<\beta$. Thus we cannot have $\gamma<\beta$ by the first part of Theorem A with $A(x)=M_{\mathcal{P}}(x)$; i.e. $\gamma=\beta$.

Thus if $\alpha \neq \beta$, then $\gamma=\Theta$. Hence the two largest numbers are always equal. Finally, since $\max \{\alpha, \beta\} \geq \frac{1}{2}$, we see that in all three cases the largest pair is always at least $\frac{1}{2}$.

2. Systems with different $\alpha, \beta, \gamma$.

It is perhaps of interest to see if it really is possible that each of $\alpha, \beta$ or $\gamma$ can be strictly less than the other two and whether it can be less than $\frac{1}{2}$. 
(a) $\beta<\alpha=\gamma$. For $\mathcal{N}=\mathbb{N}$, we have $\beta=0$ and, under the Riemann Hypothesis, $\alpha=\gamma=\frac{1}{2}$. Unconditionally, we only have $\alpha=\gamma=\Theta$ where $\Theta=\sup \{\Re \rho: \zeta(\rho)=0\}$.

(b) $\alpha<\beta=\gamma$. In the final discussion of [6], a $g$-prime system was given with $\alpha=0$. Namely, take $p_{n}=R^{-1}(n)$, where $R$ is the strictly increasing function on $[1, \infty)$ defined by

$$
R(x)=\sum_{k=1}^{\infty} \frac{(\log x)^{k}}{k ! k \zeta(k+1)}
$$

where $\zeta(\cdot)$ is the Riemann zeta-function. As such, one has $\psi_{\mathcal{P}}(x)=x+O(\log x \log \log x)$. By Theorem $1, \beta=\gamma$, but what this common value is is not clear, except that it lies in $\left[\frac{1}{2}, 1\right]$.

(c) $\gamma<\alpha=\beta$. For this we can use the example $\mathcal{P}=\mathbb{P} \sqcup \mathbb{P}^{1 / \beta}$ with $\beta \in(0,1)$. Using Dirichlet's hyperbola method, we have

$$
N_{\mathcal{P}}(x)=\sum_{m n^{1 / \beta} \leq x} 1=\zeta\left(\frac{1}{\beta}\right) x+\zeta(\beta) x^{\beta}+O\left(x^{\frac{\beta}{1+\beta}}\right)
$$

(see [7] where this calculation was done). Furthermore, $\psi_{\mathcal{P}}(x)=\psi(x)+\psi\left(x^{\beta}\right)=x+x^{\beta}+$ $O\left(x^{\frac{1}{2}+\varepsilon}\right)$ on RH. Thus $\alpha=\beta$. But, with $M(x)=\sum_{n \leq x} \mu(n)$,

$$
M_{\mathcal{P}}(x)=\sum_{m n^{1 / \beta} \leq x} \mu(m) \mu(n)=\sum_{n \leq a^{\beta}} M\left(\frac{x}{n^{1 / \beta}}\right)+\sum_{n \leq b} M\left(\left(\frac{x}{n}\right)^{\beta}\right)-M\left(a^{\beta}\right) M(b)
$$

for any $a b=x$. Putting $a=x^{\lambda}$ and using the bound $M(x) \ll x^{\frac{1}{2}+\varepsilon}$ gives

$$
\begin{aligned}
M_{\mathcal{P}}(x) & \ll \sum_{n \leq x^{\lambda \beta}}\left(\frac{x}{n^{1 / \beta}}\right)^{\frac{1}{2}+\varepsilon}+\sum_{n \leq x^{1-\lambda}}\left(\left(\frac{x}{n}\right)^{\beta}\right)^{\frac{1}{2}+\varepsilon}+\left(x^{\lambda \beta}\right)^{\frac{1}{2}+\varepsilon} x^{(1-\lambda)\left(\frac{1}{2}+\varepsilon\right)} \\
& \ll\left(x^{\frac{\beta}{2}+\lambda\left(1-\frac{\beta}{2}\right)}+x^{\frac{1}{2}+(1-\lambda)\left(\beta-\frac{1}{2}\right)}+x^{\frac{\lambda}{2}+(1-\lambda) \frac{\beta}{2}}\right) x^{\varepsilon} .
\end{aligned}
$$

Choosing $\lambda=\frac{\beta}{1+\beta}$ optimally shows that $M_{\mathcal{P}}(x) \ll x^{\frac{3 \beta}{2(1+\beta)}+\varepsilon}$ for all $\varepsilon>0$. Thus $\gamma \leq$ $\frac{3 \beta}{2(1+\beta)}<\beta$. Note that $\gamma \geq \frac{1}{2}$, since $\frac{1}{\zeta_{\mathcal{P}}(s)}=\frac{1}{\zeta(s) \zeta(s / \beta)}$ has poles on the $\frac{1}{2}$-line.

\section{Open problems}

1) From (a) and (b) above we have systems with $(\alpha, \beta, \gamma)=(a, 0, a)$ and $(0, b, b)$ for some $a, b \in\left[\frac{1}{2}, 1\right]$. Can we find, unconditionally, such systems with $a<1$ and $b<1$ ?

2) In (c) above we have a system, conditional on $\mathrm{RH}$, with $(\alpha, \beta, \gamma)=(c, c, d)$ with $\frac{1}{2} \leq d<c<1$. Can we find one unconditionally, with $d<1$. Furthermore, can we find one with $d<\frac{1}{2}$ ?

\section{References}

[1] A. Beurling, Analyse de la loi asymptotique de la distribution des nombres premiers généralisés, I, Acta Math. 68 (1937) 255-291.

[2] P.T. Bateman and H. G. Diamond, Asymptotic distribution of Beurling's generalised prime numbers in: Studies in Number Theory 6, Prentice-Hall, (1969), 152-212.

[3] G. Debruyne, H. G. Diamond and J. Vindas, $M(x)=o(x)$ estimates for Beurling numbers, J. Théor. Nombres Bordeaux 30 (2018), 469483. 
[4] G. Debruyne, F. Maes and J. Vindas, Halász's Theorem for Beurling's generalized numbers, to appear in Acta Arith., see arXiv:1902.03870.

[5] H.G. Diamond and W.-B. Zhang, Beurling Generalized Numbers, AMS Mathematical Surveys and Monographs, Vol. 213, 2016.

[6] T.W. Hilberdink, Well-behaved Beurling primes and integers, J. Number Theory 112 (2005) 332-344.

[7] T.W. Hilberdink, A lower bound for the Lindelöf function associated to generalized integers, $J$. Number Theory 122 (2007) 336-341.

[8] T.W. Hilberdink and M. L. Lapidus, Beurling zeta functions, Generalised Primes, and Fractal Membranes, Acta. Appl. Math., 94 (2006) 21-48.

[9] J.-P. Kahane et E. Saias, Fonctions complètement multiplicatives de somme nulle, Expo. Math. 35 (2017) 364-389.

[10] W.-B. Zhang, A generalization of Halász's Theorem to Beurling's generalized integers and its application, Illinois J. Math., 31 (1987) 645-664.

[11] W.-B. Zhang, Beurling primes with RH, Beurling primes with large oscillation, Math. Ann., 337 (2007) 671-704. 